\title{
El grado de alfabetización digital en el profesorado de inglés en Educación Primaria
}

\author{
The level of digital literacy in English teacher in Primary Education
}

Aurora Forteza Martínez.

Universidad de Huelva.

aurora.forteza@alu.uhu.es

Patricia de Casas Moreno.

Universidad de Nebrija. pcasas@nebrija.es

Arantxa Vizcaíno Verdú. Universidad de Huelva. arantxa.vizcaino935@alu.uhu.es

\section{RESUMEN.}

La inclusión de las Tecnologías de la Información y la Comunicación (TIC) en la vida diaria de las personas, así como su influencia en el desarrollo de los múltiples ámbitos profesionales y personales, ha cobrado un rol notorio para la sociedad a la hora de estar comunicados e informados. Debido a ello, la educación debe dar respuesta a las necesidades y realidades que existen en la actualidad y ofrecer a los alumnos una educación de calidad. De este modo, los actuales profesionales de la educación deben estar formados y actualizados, con el fin de cumplir y atender las necesidades de los estudiantes en el nuevo entorno mediático y digital. El presente estudio tiene como objetivo conocer el grado de alfabetización digital, así como los instrumentos utilizados por parte de los docentes de inglés en la etapa de Educación Primaria. Para ello, se ha llevado a cabo una investigación a través de una metodología cuantitativa con la participación de un total de 112 educadores de la especialidad de inglés de la Comunidad de Madrid, a través de la creación de una encuesta. Entre los resultados más destacados se puede señalar que los docentes apuestan por el uso de las TIC en sus aulas. Sin embargo, presentan una formación media-baja en la materia. En conclusión, hay que destacar que el nivel de alfabetización digital entre el personal docente debe ser lo más óptimo posible, ofreciendo, de ese modo, una respuesta acorde a la demanda de la sociedad.

\section{PALABRAS CLAVE.}

Alfabetización digital, docentes, educación primaria, TIC.

\section{ABSTRACT.}

The inclusion of Information and Communication Technologies (ICT) in people's daily lives, as well as their influence on the development of multiple professional and personal spheres, has taken on a notorious role for society when it comes to being communicated and informed. This is leading to the creation of numerous jobs that, until a few years ago, did not exist. Because of this, education must respond to the needs and realities that exist today and offer students a quality education. In this way, current education professionals must be trained and updated in order to meet and attend to the needs of students in the new media and digital

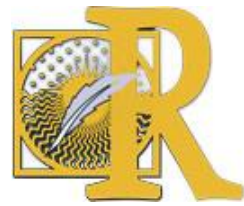

Fecha de recepción: 26-04-2019 Fecha de aceptación: 24-10-2019

Forteza, A., De Casas, P. \& Vizcaíno, A. (2020). El grado de alfabetización digital en el profesorado de inglés 
environment. The objective of this study is to know the degree of digital literacy, as well as the instruments used by English teachers in the Primary Education stage. To this end, research has been carried out using a quantitative methodology with the participation of a total of 112 educators specialising in English from the Community of Madrid, through the creation of a survey. Among the most outstanding results, it can be pointed out that teachers are committed to the use of ICT in their classrooms. However, they present a medium-low training in the subject. In conclusion, it should be pointed out that the level of digital literacy among teaching staff must be as optimal as possible, thus offering a response in accordance with society's demand.

\section{KEY WORDS.}

Digital literacy, teachers, primary school, ICTs.

\section{Introducción.}

La irrupción de las Tecnologías de la Información y la Comunicación (TIC) han provocado que estas se conviertan en una parte integrante y fundamental de la vida de las personas y la sociedad en general. Esta realidad plantea nuevas formas de concebir el sistema educativo, donde la inclusión de las TIC plantea nuevos retos y horizontes para los profesionales de la educación, viendo la necesidad de estar adecuadamente formados para poder transmitir una educación de calidad basada en dispositivos tecnológicos.

Es conveniente hacer mención a la implicación que tienen las nuevas generaciones en el uso y disfrute de estas tecnologías, teniendo cada vez más una presencia notable en sus vidas. El primer gran referente en aportar una definición a este concepto fue Prensky (2011), el cual afirmó que un nativo digital es aquel individuo nacido a partir del año 1990 y que, como consecuencia de sus experiencias y prácticas relacionadas con diversos dispositivos, como el ordenador o el teléfono móvil, son verdaderos expertos en la materia, reuniendo una serie de destrezas que le permiten realizar numerosas y diversas tareas sin que ello conlleve ningún esfuerzo.

Tal y como apunta Hernández (2017), que el uso de dispositivos tecnológicos se lleva empleando desde hace varias décadas. Tal es el caso del uso de la calculadora, la televisión y el retroproyector. Por ello, el punto de vista del autor radica en la necesidad que existe en la actualidad de formar adecuadamente al docente. Igualmente, es importante reforzar esta formación dentro de la materia de competencia digital con el fin de dar respuesta y atender de manera óptima al alumnado ante la realidad existente en el mundo actual. Sin embargo, de acuerdo a lo expuesto por Arrieta y Montes (2011), a pesar de la aceptación de la importancia de la inclusión de las TIC dentro del aula y los beneficios que ello conlleva para el proceso de enseñanza-aprendizaje en los alumnos, son muchos los educadores que muestran su falta de compromiso para incorporarlas en su práctica diaria habitual.

En definitiva, la presente investigación trata de dar respuesta a cuál es el uso real que se hace de las TIC dentro del aula de Educación Primaria y, más detalladamente, entre los docentes del área de inglés.

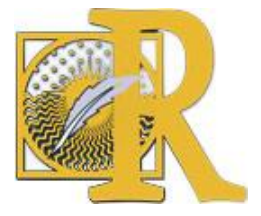

Fecha de recepción: 26-04-2019 Fecha de aceptación: 24-10-2019 Forteza, A., De Casas, P. \& Vizcaíno, A. (2020). El grado de alfabetización digital en el profesorado de inglés en Educación Primaria 


\section{Marco teórico.}

\subsection{La alfabetización digital en la educación.}

La inclusión de las TIC permite el acceso a una cantidad ingente de información, siendo necesaria una correcta formación en este ámbito con el fin de poder obtener el máximo provecho de los recursos digitales. Todo ello ha generado la necesidad de una formación específica para utilizar de manera óptima dichos medios, término acuñado como alfabetización digital. El afán de erradicar el analfabetismo tradicional a nivel mundial, da lugar a que en la actualidad se considere prioritaria una correcta alfabetización digital, quedando constancia de ello en la "Carta para la alfabetización de los medios" (2006), donde se refleja la importancia de la formación a nivel mundial dentro de este ámbito.

Este término de alfabetización digital no es un concepto reciente, ya que se han llevado a cabo múltiples estudios en torno a él. En relación a ello, es conveniente mencionar la exposición que hace Gilbert (1997), aludiendo a la competencia que las personas poseen para poder entender, así como utilizar los diferentes recursos y dispositivos tecnológicos.

Hay que destacar, en el caso concreto de España, tal y como afirman Sánchez, Ramos y Sánchez (2014) en las últimas décadas se ha apuntado, desde las diferentes legislaciones educativas, la necesidad de que los profesionales de la educación posean un correcto aprendizaje dentro del terreno de las TIC, siendo esta introducida en los programas de estudios. En este sentido, Cabero (2016) hace referencia a la utilización de nuevas denominaciones para referirse a las generaciones de alumnos, es decir, al denominarlos como nativos digitales. Por su parte, Lorenzo (2005) acuña el término de generación red, caracterizados por ser expertos en aprender a aprender, ya que desde muy jóvenes han adquirido el conocimiento pertinente en torno a las TIC.

En definitiva, bajo esta nueva realidad se deben asimilar por parte de los educadores, nuevos perfiles adecuados a la sociedad en la que se está desempeñando su labor profesional. El profesor se ha convertido en una herramienta que refuerza la creación de nuevas situaciones de aprendizaje y metodologías, donde el protagonismo recae en la figura del estudiante. Todo esto va a suponer la creación de recursos y materiales adecuados a las nuevas situaciones que existen dentro del aula (Castañeda, 2017; Luz, 2018).

\subsection{El contexto educativo y la inclusión de las TIC.}

El cambio que se ha producido en la sociedad en las últimas décadas con la inclusión de las TIC ha sido incuestionable, afectando a todos los aspectos de los ámbitos social, económico y tecnológico. Por ende, la educación debe dar una solución a los posibles interrogantes o dilemas que de esta nueva estructura surja. Es necesario formar a los alumnos en una correcta competencia digital, así como prepararles para el uso de diferentes herramientas y recursos disponibles en Internet. Al hablar de competencia digital, es necesario hacer mención a la incorporación de las TIC dentro del aula, lo cual supone una repercusión directa dentro del proceso de enseñanza-aprendizaje de los estudiantes (Plaza de la Hoz, 2017). Según Coll, Mauri y Onrubia (2008), aseguran que las TIC ofrecen numerosos y diversos beneficios, entre los que se encuentran el seguimiento y control de los diferentes avances, así como los obstáculos que los estudiantes obtienen, ofreciendo la posibilidad al docente de

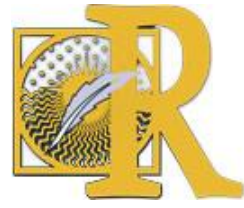

Fecha de recepción: 26-04-2019 Fecha de aceptación: 24-10-2019

Forteza, A., De Casas, P. \& Vizcaíno, A. (2020). El grado de alfabetización digital en el profesorado de inglés 
ofrecer soporte en los diferentes momentos del proceso de aprendizaje. Todas estas afirmaciones están sustentadas por lo expuesto en las leyes educativas españolas Ley Orgánica de Educación (LOE, 2006), Ley Orgánica para la Mejora de la Calidad Educativa (LOMCE, 2013) y Ley Orgánica de Ordenación General del Sistema Educativo (LOGSE, 1990), donde ya en los preámbulos se expresa la necesidad de regenerar el sistema educativo español actual, lo que implica que el personal docente posea una buena formación en los diferentes ámbitos de su práctica, siendo uno de ellos el campo de las TIC, como consecuencia de la sociedad del conocimiento en la que se vive en la actualidad.

Múltiples autores e investigadores aluden a la importancia de tener un programa adecuado a las necesidades formativas de los profesores dentro de la materia de la competencia digital (Sangrá, 2016; Llamas-Salguero y Macías Gómez, 2017). Gutiérrez (2008) apunta a que una correcta formación no depende única y exclusivamente de la formación teórica, ya que se requiere, además, de una buena cultura práctica y didáctica. Por ello, dentro de los estudios que los docentes realizan, es imprescindible que exista una enseñanza basada en la competencia digital, la cual debe estar fundamentada en el uso adecuado de las TIC (Porlán, Espinosa y Sánchez, 2018). Para ello, todos y cada uno de los profesores deben de ser conscientes de la repercusión y transcendencia que, a día de hoy, poseen los recursos e instrumentos digitales en una sociedad inmersa en la era digital, posibilitando la integración de estas herramientas dentro de la vida diaria de las escuelas y haciendo a los estudiantes partícipes de las tareas que se desarrollen (Barrantes, Casas y Luengo, 2011)

Por otro lado, Cassany y Ayala (2008) explican que los jóvenes están formándose de una manera distinta a lo que ocurría hace dos décadas, realizando un aprendizaje de diferentes destrezas y habilidades de una manera informal, sin que ellos sean conscientes de esta situación. De acuerdo con lo expuesto por el informe del Programa Internacional para la Evaluación de Estudiantes (PISA) (OECD, 2015), cabe apuntar que, el rendimiento educativo y formativo de los alumnos con el uso de los dispositivos tecnológicos, después de la dotación que han realizado diferentes países para la implantación de estos recursos dentro de las aulas, no ha incrementado los niveles de rendimiento en materias como lectura, matemáticas o ciencia.

\section{Metodología.}

\subsection{Contexto.}

El presente estudio tiene como objetivo principal conocer el grado de alfabetización digital, así como los instrumentos utilizados por parte de los docentes de inglés en la etapa de Educación Primaria. Asimismo, también se han atendido a una serie de objetivos específicos como descubrir el nivel formativo de los mismos, conocer qué herramientas y recursos digitales emplean, la incorporación de los recursos tecnológicos en sus aulas, y mostrar la importancia que tienen para ellos las TIC en los procesos de enseñanza.

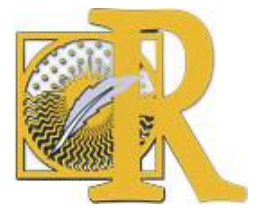

Fecha de recepción: 26-04-2019 Fecha de aceptación: 24-10-2019 


\subsection{Diseño e instrumentos de investigación.}

Para llevar a cabo la investigación, se ha realizado un estudio basado en metodología cuantitativa a través del uso de encuestas como método de análisis. Dicho instrumento ha sido validado por un comité de expertos, tanto nacionales como internacionales, dentro del ámbito de estudio. Según Cabero y Llorente-Cejudo (2013), es importante solicitar a una serie de personas un juicio planteado sobre un objeto y su finalidad en sí mismo. En este sentido, para medir el grado de acuerdo entre los expertos consultados, se ha empleado el programa estadístico SPSS con el fin de revisar si la medida de acuerdo es alta, estableciéndose un consenso en el proceso de valoración y una mayor posibilidad de réplica del instrumento de medición.

Tras realizar las pruebas de fiabilidad del instrumento se ha confirmado el grado de consistencia interna con un Alpha de Cronbach de 0.982. La encuesta ejecutada se encuentra conformada por un total de 76 ítems y está integrada por tres bloques diferenciados: en primer lugar, el perfil sociodemográfico, donde se atiende al perfil de los encuestados; en segundo lugar, se profundiza en el conocimiento y uso de las TIC por parte de la muestra del estudio; y el último bloque realiza la exposición de múltiples premisas relacionadas con el análisis de la alfabetización digital, competencias y habilidades en el uso de las TIC en el aula de inglés, las cuales son evaluadas a través de una escala Likert de 1 a 5.

\subsection{Participantes del estudio.}

Una vez obtenida la fiabilidad del instrumento, se llevó a cabo la recogida de datos con la colaboración de la muestra seleccionada. En este proceso de investigación han participado un total de 112 docentes, correspondientes a la especialidad de inglés y trabajando dentro de la Comunidad Autónoma de Madrid. De este modo, hay que destacar que se trata de estudio exploratorio y piloto, y por ello, se ha atendido a una muestra intencional con el fin de que todas aquellas personas encuestadas fueran de la especialidad de inglés $y$, por otro lado, aleatoria, porque no se ha tenido en cuenta su ubicación dentro del área de la Comunidad de Madrid, tipo de centro u otro tipo de característica.

\section{Resultados.}

\subsection{Perfil socio-demográfico.}

En lo relativo a la edad de la muestra de estudio, se puede advertir que el grupo mayoritario oscila entre los 30 y los 35 años de edad (32\%), siendo el rango de edad de 50 en adelante el de menor participación (5\%). Asimismo, en lo que respecta al género de los encuestados, se observa que la mayoría de ellos son mujeres (86\%), frente a los hombres (14\%).

Por otro lado, en lo referente al tiempo que los docentes llevan trabajando, el rasgo más común se sitúa en la franja de 0 a 5 años (37\%), seguido de aquellos que llevan entre 5 y 10 años (36\%).

Por último, cabe destacar que la mayoría de los encuestados trabaja en un centro educativo de titularidad pública (80\%), sobre los concertados (16\%) y privados $(4 \%)$.

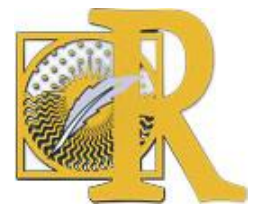

Fecha de recepción: 26-04-2019 Fecha de aceptación: 24-10-2019 


\subsection{Conocimiento y uso de las TIC.}

Para poder llevar a cabo el estudio, se consultó a los encuestados sobre qué tipo de formación poseen los docentes dentro del ámbito de las Tecnologías, cuáles son los dispositivos que poseen, así como dónde y cuándo se utilizan. Del mismo modo, se analizan cuáles son los programas y aplicaciones que emplean en su vida personal como medio de comunicación, así como analizar la frecuencia con la que manejan las herramientas tecnológicas dentro del aula. Por tanto, es necesario conocer la opinión que tienen los docentes sobre el uso de las mismas con los alumnos en el aula, así como las principales competencias digitales, que debe tener un docente y, como consecuencia, sobre qué áreas desearían poseer más formación.

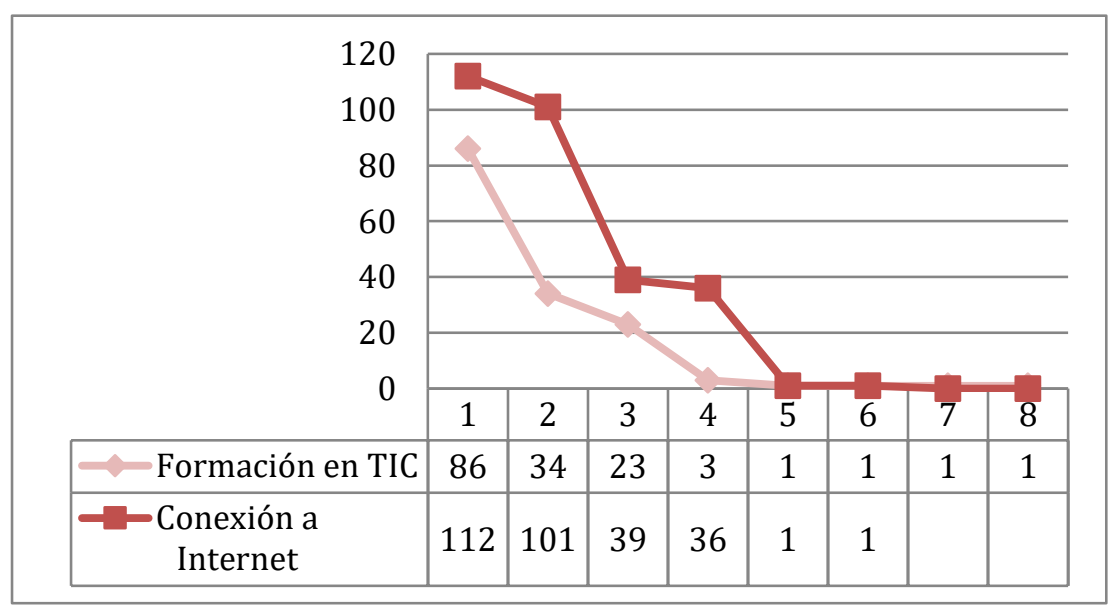

Figura 1. Formación en TIC y conexión a Internet.

Fuente: Elaboración propia a partir de los datos obtenidos.

En relación a la formación académica que los educadores encuestados reconocen tener, sobresale la opción de cursos de formación (86) sobre las demás, pudiéndose observar cómo tan solo 23 de los encuestados posee un máster específico sobre la materia. Además, con respecto a los lugares donde los docentes se conectan a Internet, se muestra como la totalidad de ellos (112) lo hace desde sus domicilios. También, se puede apreciar como una gran mayoría (101) lo hace cuando se encuentra en su puesto de trabajo. Por último, al analizar cuánto tiempo dedican al uso de Internet diariamente por parte de los docentes, se puede percibir como algo más de la mitad de la muestra (67) realiza un uso moderado, el cual oscila entre una y tres horas al día. En el menor de los casos (9), se admite que su empleo supera las seis horas a lo largo del día (Figura 1).

Por otro lado, atendiendo al manejo de los diferentes dispositivos tecnológicos y, a su vez, el disfrute de las múltiples aplicaciones y programas por parte de los encuestados, se puede incidir en los siguientes resultados (Figura 2):

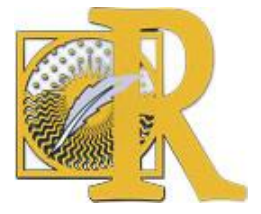

Fecha de recepción: 26-04-2019 Fecha de aceptación: 24-10-2019 


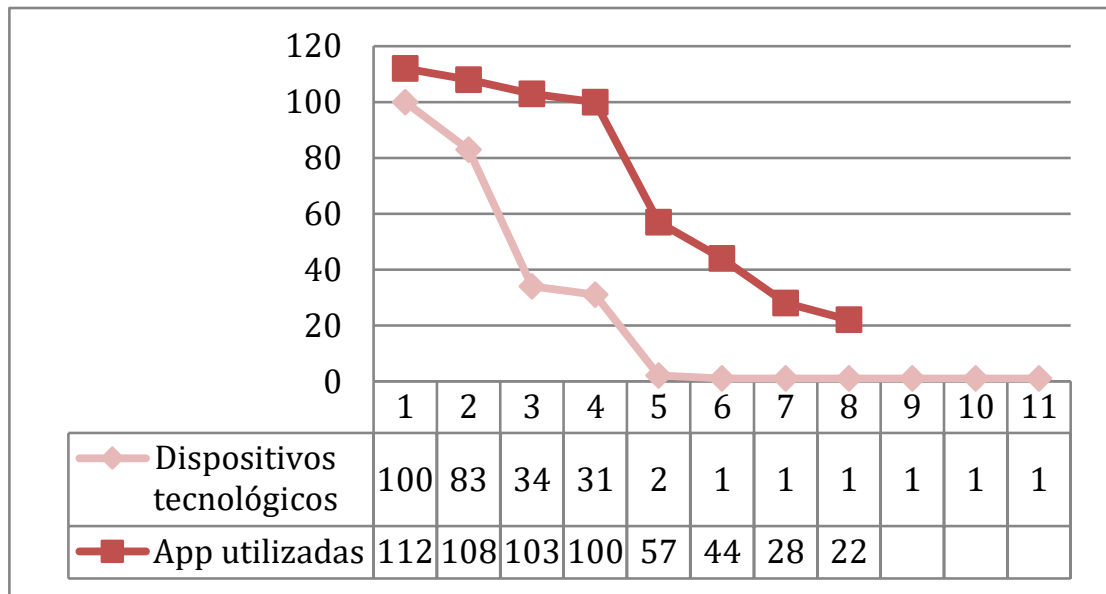

Figura 2. Dispositivos tecnológicos y App utilizadas.

Fuente: Elaboración propia a partir de los datos obtenidos.

Al tratar los dispositivos tecnológicos de los que dispone la población, destaca el Smartphone (100), siendo este dispositivo uno de los que más ha evolucionado en las últimas décadas, implantándose en la vida de las personas. Además, el segundo dispositivo electrónico más utilizado por los encuestados es la Tablet (83), estando cada vez más integrada en las actividades cotidianas de los usuarios. Asimismo, se puede confirmar que casi la totalidad de la población encuestada (110) reconoce utilizar estos dispositivos en el hogar. Sin embargo, una gran mayoría (93) afirma que su uso también lo realiza durante su jornada laboral cuando está en el centro educativo.

Entre los programas y aplicaciones más populares manejados por los profesionales de la educación en su vida diaria, se observa que la totalidad de los participantes en el estudio (112) utilizan WhatsApp, siendo esta aplicación la más conocida y utilizada en la actualidad por la población. Dentro de las redes sociales, cabe destacar Facebook como la más popular entre el profesorado (103). También, cabe señalar como el uso de Messenger, una plataforma de comunicación entre los usuarios de la misma, fue muy popular entre finales de la década de los 90 y principios del mileno, a día de hoy no es programa muy utilizado entre la población; de hecho, tan solo 22 de los participantes admite seguir utilizándola.

Una vez estudiado el uso a nivel personal que los docentes realizan de las TIC, la investigación continúa examinando qué uso se realiza dentro de las aulas de los recursos tecnológicos existentes. Una gran mayoría (72) reconoce que hace un uso diario de los mismos, y una minoría (8) admite no utilizarlos nunca. Mientras que un total de 32 sujetos señalan su aprovechamiento con frecuencia.

Por otro lado, al preguntar a los docentes por la opinión que estos tienen sobre las TIC dentro del aula, una gran representación (99) expresa que son una herramienta que fomenta la motivación entre los alumnos, siempre y cuando este uso sea adecuado y responsable, según lo afirman 91 de los participantes del estudio. Muchos de los sujetos de la muestra (77) pronuncian su convicción que, para que un proyecto digital en los centros educativos sea adecuado y tenga éxito, es imprescindible un cambio metodológico.

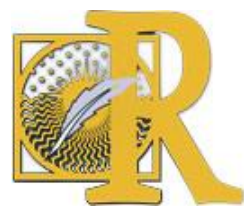

Fecha de recepción: 26-04-2019 Fecha de aceptación: 24-10-2019 
En relación a la posibilidad que ofrecen los recursos digitales para adaptar las múltiples actividades planteadas a los estudiantes, algo más de la mitad de los encuestados (58) expresa su conformidad con esto. No obstante, se puede apreciar cómo existen reticencias a la hora de asegurar el éxito de una clase cuando se utilizan las TIC (24). Cabe señalar que una minoría (12) expresa su conformidad en cuanto a la adecuación y optimización de la existencia de recursos digitales en los centros educativo y, por otro lado, es llamativo que tan solo 4 sujetos afirmen que la formación docente, a día de hoy con respecto al área de las $\mathrm{TIC}$, es inmejorable.

Continuando con esta línea temática, hay que destacar que, al hablar de las competencias digitales, los educadores deben poseer una metodología basada en las TIC para poder trabajar dentro de las aulas. El análisis de resultados indica que es uno de los términos más importantes considerado por los participantes en el estudio (95). Además, se hace referencia al uso de contenidos digitales con los alumnos, así como un adecuado conocimiento de las múltiples aplicaciones (84). Cabe resaltar que el uso de las redes sociales como vía de comunicación entre los profesores, por un lado, y los alumnos y/o familias por otro, aún genera cierta incertidumbre y desconfianza entre los docentes, ya que tan solo 18 de ellos apuesta por esta vía como medio de intercambio de información.

Para finalizar con este bloque, se plantearon una serie de opciones a los encuestados referentes a los múltiples ámbitos de formación, donde les gustaría seguir aprendiendo y profundizando sus conocimientos, destrezas y habilidades. Entre las diferentes alternativas expuestas, cabe destacar el interés de este colectivo por el área de gamificación y juegos digitales (74), seguido por la alternativa de creación de contenidos (73). En referencia al resto de los ámbitos de formación se encuentra el uso de aplicaciones y la pizarra digital, ambas con un total de 54 sujetos, seguido de la narrativa digital con 36 , la realidad aumentada con 33 , la realidad virtual con 29 , las redes sociales con 18 y el aprendizaje móvil con 14 (Figura 3).

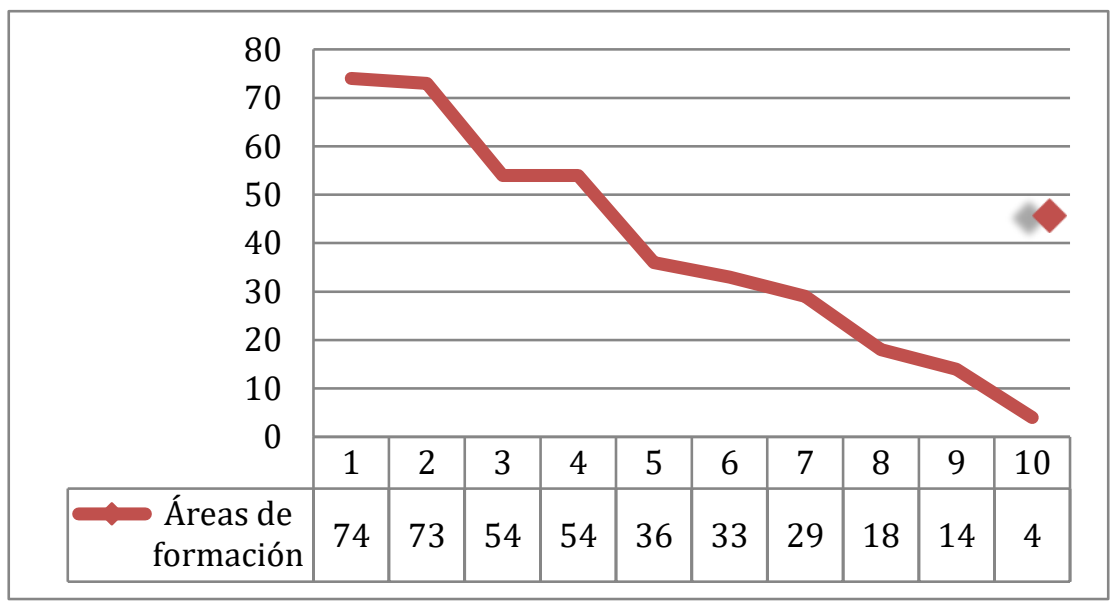

Figura 3. Áreas de formación deseadas entre los docentes. Fuente: Elaboración propia a partir de los datos obtenidos.

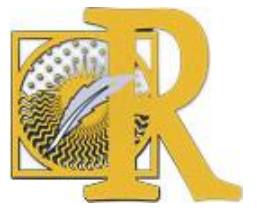

Fecha de recepción: 26-04-2019 Fecha de aceptación: 24-10-2019 


\subsection{Alfabetización digital, competencias y habilidades en el uso de las TIC en el aula de inglés.}

Para analizar el nivel de alfabetización digital que los docentes del área de inglés de la Comunidad de Madrid poseen, así como las diferentes competencias y habilidades que del uso de las TIC se desprenden, se han analizado los resultados a través de la media y la desviación típica de las respuestas.

Tabla 1. Opinión de los docentes en relación a las TIC.

\begin{tabular}{lrrrrr}
\hline & $\mathrm{N}$ & Mínimo & Máximo & Media & Desv. típ. \\
Item 17 & 112 & 1 & 5 & 4,35 & 1,011 \\
Item 18 & 112 & 2 & 5 & 4,22 &, 835 \\
Item 19 & 112 & 1 & 5 & 4,08 &, 960 \\
Item 20 & 112 & 2 & 5 & 4,13 &, 902 \\
Item 21 & 112 & 2 & 5 & 4,33 &, 884 \\
Item 22 & 112 & 2 & 5 & 4,22 &, 908 \\
Item 23 & 112 & 2 & 5 & 3,92 &, 978 \\
Item 24 & 112 & 1 & 5 & 3,04 &, 934 \\
Item 25 & 112 & 2 & 5 & 3,88 &, 908 \\
Item 26 & 112 & 1 & 5 & 2,48 & 1,237 \\
Item 27 & 112 & 1 & 5 & 4,25 & 1,061 \\
N válido (según lista) & 112 & & & & \\
\hline
\end{tabular}

Fuente: Elaboración propia a partir de los datos obtenidos de SPSS.

Para comenzar con el análisis sobre la opinión que tienen los docentes de las TIC, se aprecia cómo la media presenta valores cercanos a 4, y la desviación típica gira en torno a 1, por lo que no existen diferencias estadísticas significativas (Tabla 1). En lo que respecta a los valores más elevados, cabe mencionar los ítems $17(4,35), 21(4,33)$ y $27(4,25)$, los cuales hacen referencia a "Una buena formación en TIC implica estar actualizado", "Fomentan la motivación del alumnado para aprender inglés" e "Implica una buena dotación en las aulas de recursos tecnológicos". Por otro lado, los elementos cuyos valores son inferiores hacen alusión a los ítems $26(2,48), 24(3,04)$ y $25(3,88)$, los cuales corresponden a las siguientes afirmaciones: "Es complicado su uso con niños pequeños", "Suponen una mayor implicación de las familias" y "Su uso en el aula implica más beneficios que desventajas".

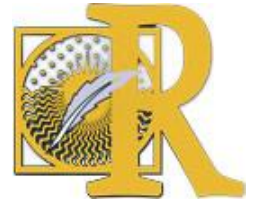

Fecha de recepción: 26-04-2019 Fecha de aceptación: 24-10-2019 
Tabla 2. Uso de las TIC dentro del aula de inglés.

\begin{tabular}{lrrrrr}
\hline & $\mathrm{N}$ & Mínimo & Máximo & Media & Desv. típ. \\
Item 28 & 112 & 1 & 5 & 4,29 & 1,045 \\
Item 29 & 112 & 1 & 5 & 4,27 & 1,057 \\
Item 30 & 112 & 1 & 5 & 4,10 & 1,048 \\
Item 31 & 112 & 1 & 5 & 3,84 & 1,249 \\
Item 32 & 112 & 1 & 5 & 3,49 & 1,280 \\
Item 33 & 112 & 1 & 5 & 4,05 & 1,056 \\
Item 34 & 112 & 1 & 5 & 3,45 & 1,387 \\
N válido (según lista) & 112 & & & & \\
\hline
\end{tabular}

Fuente: Elaboración propia a partir de los datos obtenidos de SPSS.

En un segundo apartado, en referencia al uso que se hace de las TIC dentro del aula con los alumnos, la desviación típica ofrece valores cercanos a 1, por lo que no se aprecian valores estadísticos significativos (Tabla 2). En relación a la meda, los valores se sitúan en torno a 4, concurriendo los ítems mejor valorados el 28 (4,29), $29(4,27)$ y $30(4,10)$, estas afirmaciones las siguientes: "Ver vídeos en inglés", "Escuchar canciones en inglés" y "Ejercicios interactivos relacionados con la asignatura". Sin embargo, los ítems con menor media son el $34(3,45), 32(3,49)$ y $31(3,84)$, los cuales expresan "Leer cuentos interactivos", "Utilizar aplicaciones" y "Buscar información en Internet".

Tabla 3. Conocimiento de herramientas TIC.

\begin{tabular}{lrrrrr}
\hline & $\mathrm{N}$ & Mínimo & Máximo & Media & Desv. típ. \\
Item 35 & 112 & 1 & 5 & 1,44 & 1,029 \\
Item 36 & 112 & 1 & 5 & 2,73 & 1,687 \\
Item 37 & 112 & 1 & 5 & 3,11 & 1,618 \\
Item 38 & 112 & 1 & 5 & 2,51 & 1,525 \\
Item 39 & 112 & 1 & 5 & 3,13 & 1,636 \\
Item 40 & 112 & 1 & 5 & 2,54 & 1,671 \\
Item 41 & 112 & 1 & 5 & 1,75 & 1,270 \\
Item 42 & 112 & 1 & 5 & 2,31 & 1,617 \\
Item 43 & 112 & 1 & 5 & 1,50 &, 995 \\
Item 44 & 112 & 1 & 5 & 3,34 & 1,631 \\
Item 45 & 112 & 1 & 5 & 2,39 & 1,618 \\
Item 46 & 112 & 1 & 5 & 1,84 & 1,305 \\
Item 47 & 112 & 1 & 5 & 3,37 & 1,622 \\
Item 48 & 112 & 1 & 5 & 2,06 & 1,397 \\
Item 49 & 112 & 1 & 5 & 3,21 & 1,639 \\
Item 50 & 112 & 1 & 5 & 3,55 & 1,615 \\
Item 51 & 112 & 1 & 5 & 1,96 & 1,395 \\
Item 52 & 112 & 1 & 5 & 3,60 & 1,467 \\
N válido (según lista) & 112 & & & & \\
\hline
\end{tabular}

Fuente: Elaboración propia a partir de los datos obtenidos de SPSS.

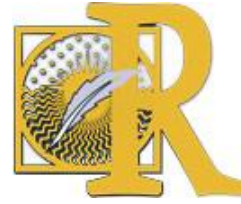

Fecha de recepción: 26-04-2019 Fecha de aceptación: 24-10-2019 
En este tercer apartado, se analiza el conocimiento que tienen los docentes de las diferentes herramientas relacionadas con las TIC (Tabla 3). Al atender a la desviación típica los valores son cercanos a 1, por lo que no existen diferencias estadísticas significativas. En referencia a la media, se aprecian valores dispares entre las afirmaciones. Los ítems que muestran una valoración más alta son el $52(3,60), 50(3,55)$ y $47(3,37)$, siendo las siguientes herramientas GoogleApps, Blogger y ClassDojo. Sin embargo, los ítems con un índice de media más bajo son el $35(1,44), 43(1,50)$ y $41(1,75)$, haciendo alusión a Zoho, Symphonical y Mindmeister. Del análisis de esta pregunta se puede apreciar cómo no poseen un igual conocimiento de las diferentes herramientas que existen en la actualidad para su uso dentro del aula de inglés.

Tabla 4. Las redes sociales en el aula de inglés.

\begin{tabular}{lrrrrr}
\hline & $\mathrm{N}$ & Mínimo & Máximo & Media & Desv. típ. \\
Item 53 & 112 & 1 & 5 & 1,55 &, 985 \\
Item 54 & 112 & 1 & 5 & 1,81 & 1,212 \\
Item 55 & 112 & 1 & 5 & 1,67 & 1,118 \\
Item 56 & 112 & 1 & 5 & 4,21 & 1,150 \\
Item 57 & 112 & 1 & 5 & 3,39 & 1,538 \\
Item 58 & 112 & 1 & 5 & 1,50 &, 995 \\
Item 59 & 112 & 1 & 5 & 3,61 & 1,435 \\
Item 60 & 112 & 1 & 5 & 3,23 & 1,507 \\
Item 61 & 112 & 1 & 5 & 2,46 & 1,394 \\
N válido (según lista) & 112 & & & & \\
\hline
\end{tabular}

Fuente: Elaboración propia a partir de los datos obtenidos de SPSS.

En el apartado relacionado a qué redes sociales utilizarían como medio de comunicación en el aula de inglés con alumnos y familias, se aprecia que los valores referentes a la desviación típica son cercanos a 1. De este modo, no existen diferencias estadísticas significativas (Tabla 4). En referencia a la media, los que presentan una mayor media son los ítems 56 $(4,21), 59(3,61)$ y $57(3,39)$, haciendo alusión a YouTube, Pinterest y Spotify. Sin embargo, los ítems con menor valoración son el $58(1,50), 53(1,55)$ y $55(1,67)$, siendo estas redes sociales WhatsApp, Facebook e Instagram. 
Tabla 5. Aplicaciones de inglés conocidas por los docentes.

\begin{tabular}{lrrrrr}
\hline & $\mathrm{N}$ & Mínimo & Máximo & Media & Desv. típ. \\
Item 62 & 112 & 1 & 5 & 2,71 & 1,326 \\
Item 63 & 112 & 1 & 5 & 2,91 & 1,359 \\
Item 64 & 112 & 1 & 5 & 3,20 & 1,394 \\
Item 65 & 112 & 1 & 5 & 2,84 & 1,379 \\
Item 66 & 112 & 1 & 5 & 3,06 & 1,410 \\
Item 67 & 112 & 1 & 5 & 3,73 & 1,414 \\
Item 68 & 112 & 1 & 5 & 3,03 & 1,332 \\
Item 69 & 112 & 1 & 5 & 3,06 & 1,410 \\
Item 70 & 112 & 1 & 5 & 2,73 & 1,273 \\
Item 71 & 112 & 1 & 5 & 2,76 & 1,317 \\
Item 72 & 112 & 1 & 5 & 2,80 & 1,361 \\
Item 73 & 112 & 1 & 5 & 2,81 & 1,339 \\
Item 74 & 112 & 1 & 5 & 2,81 & 1,353 \\
N válido (según lista) & 112 & & & & \\
\hline Fuent: Elaboracion propia
\end{tabular}

Fuente: Elaboración propia a partir de los datos obtenidos de SPSS.

Por último y, para concluir este proceso, se requiere conocer cuáles son las aplicaciones educativas asociadas a la enseñanza del inglés que más conocen y utilizan los profesionales en educación de esta asignatura (Tabla 5). La desviación típica presenta valores cercanos a 1 , por lo que no existen diferencias estadísticas significativas y la media se sitúa en torno a 3. Dentro de las aplicaciones con una media más elevada, destacan los ítems $67(3,73), 64$ $(3,20)$ y con la misma media los ítems 66 y 69 (3,06); los cuales hacen alusión a las siguientes plataformas: Learn English for kids, Quizlet, y ARFlashcards y My life in English. En el lado opuesto, los ítems con una media más baja son el $62(2,71), 70(2,73)$ y $71(2,76)$, haciendo referencia a las aplicaciones Roseta Stone Kids, Dic - Dic y Funland. De este estudio se desprende que el conocimiento y uso de las diferentes aplicaciones para la enseñanza del inglés no está muy extendido entre los docentes que imparten la materia.

\section{Discusión y conclusiones.}

La relevancia que tienen actualmente las TIC en todos los ámbitos de la vida es un hecho latente. Sin embargo, a pesar de la importancia que tienen para el desarrollo y beneficio de los diferentes colectivos, es un hecho que su implantación no ha llegado a todas las áreas por igual. Un claro ejemplo de ello es el terreno educativo. Tal y como señalan Fernández y Fernández (2016), uno de los principales escollos, que impiden que se lleve a cabo una correcta creación y establecimiento de estas herramientas digitales dentro de colegios e institutos depende, en gran medida, por la escasez de una formación correcta de los profesores. De hecho, del estudio llevado a cabo a través de esta investigación, se llega a la conclusión de que el nivel de formación de los docentes en el terreno de las competencias digitales es medio-bajo, debido a que no poseen los conocimientos y aptitudes apropiadas para su uso en la clase, a pesar de la importancia que desde las leyes educativas se le está

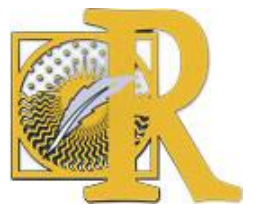

Fecha de recepción: 26-04-2019 Fecha de aceptación: 24-10-2019 
dando a las competencias digitales y mediáticas para lograr una enseñanza actualizada (Mayorga et al., 2011).

Si bien es cierto que la formación que a día de hoy presentan los docentes no es suficiente, es necesario aclarar que ha ido evolucionando con el tiempo. Atendiendo a los resultados expuestos, el $76,8 \%$ de los encuestados afirma que ha recibido cursos de formación en la materia. Sin embargo, el número desciende a medida que el nivel de estudios aumenta, donde tan solo 23 de los 112 profesionales posee un máster relacionado con este ámbito de estudios. A pesar de ello, también se ha manifestado un interés por continuar ampliando los conocimientos en diversas temáticas, como en juegos digitales y gamificación $(66,1 \%)$, creación de contenidos $(65,2 \%)$, o el uso de la pizarra digital $(48,2 \%)$, entre otros.

En lo referente a las metodologías utilizadas hoy en día, se puede comprobar una clara influencia recibida de las TIC, y así lo aprecian también los educadores, debido a que una amplia mayoría $(88,4 \%)$ afirman que su uso con los alumnos favorece la motivación para el aprendizaje si se utilizan estas herramientas en el aula. Para ello, un 81,3\% considera fundamental el uso responsable de estos recursos cuando se trabaja con niños. Sin embargo, a pesar de los beneficios que ello implica, cabe destacar que un $21,4 \%$ no relaciona el uso de las TIC con el éxito final en el proceso de enseñanza-aprendizaje.

Por otro lado, es curioso observar cómo cambia el uso de aplicaciones y herramientas en el ámbito personal y en el laboral. Claro es el ejemplo del uso de WhatsApp, ya que todos los docentes reconocen utilizar esta aplicación en su vida diaria como medio de comunicación con su red de familiares y amigos. No obstante, su uso decae en el terreno laboral con las familias como medio de relación familia-escuela, siendo la de menor uso.

Dentro del aula de inglés, el uso de dispositivos tecnológicos y herramientas digitales, así como sus aplicaciones, han hecho su irrupción. Ello conlleva que los educadores comiencen a integrarlas, de manera lenta y paulatina, en su práctica docente diaria. Los recursos que se utilizan con mayor frecuencia son, entre otros, ver vídeos en inglés de canciones o cuentos, la realización de ejercicios interactivos y juegos adecuados a las edades de los estudiantes. Asimismo, las aplicaciones son vistas como excelentes recursos que ayudan a afianzar los contenidos del idioma.

Las redes sociales también ocupan un lugar visible en la actualidad. Si bien es cierto que los docentes las utilizan en su vida personal, no gozan de tanta fama a la hora de hacerlo en el ámbito educativo. Es más, del total de encuestados, 79 desaconseja el uso de Facebook en el colegio, seguido de cerca por Instagram y Twitter. Sin embargo, la plataforma YouTube goza de buena reputación entre los docentes e inspira mayor confianza en su uso.

El estudio revela el grado de conocimiento y utilización, que, en la actualidad, se tiene de los recursos digitales por parte de los docentes en el aula de inglés, tanto a nivel personal como profesional. Después de haber analizado los resultados obtenidos en la encuesta, se ha podido comprobar cómo es necesaria una formación en este ámbito por parte de los profesionales de la educación, con el fin de poder adaptarse a las diferentes situaciones y metodologías que del uso de las TIC se desprenden, por medio de diferentes vías de formación.

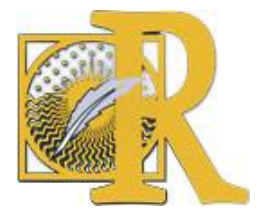

Fecha de recepción: 26-04-2019 Fecha de aceptación: 24-10-2019 Forteza, A., De Casas, P. \& Vizcaíno, A. (2020). El grado de alfabetización digital en el profesorado de inglés en Educación Primaria 
Por último, señalar dentro de las limitaciones y prospectivas de futuro que la presente investigación parte de un estudio exploratorio dentro del área de inglés. Sin embargo, se espera en un futuro poder realizar las comparativas pertinentes en las múltiples áreas académicas del currículo de Educación Primaria.

\section{Apoyos}

Este trabajo se ha elaborado en el marco de Alfamed (Red Interuniversitaria Euroamericana de Investigación en Competencias Mediáticas para la Ciudadanía), con el apoyo del Proyecto I+D+I Coordinado "Competencias mediáticas de la ciudadanía en medios digitales emergentes (smartphones y tablets): practicas innovadoras y estrategias educomunicativas en contextos múltiples" (EDU2015-64015-C3-1-R) (MINECO/FEDER), y de la "Red de Educación Mediática" del Programa Estatal de Investigación Científica-Técnica de Excelencia, Subprograma Estatal de Generación de Conocimiento (EDU2016-81772-REDT), financiados por el Fondo Europeo de Desarrollo Regional (FEDER) y Ministerio de Economía y Competitividad de España.

\section{Referencias bibliográficas.}

- Alva, A.R. (2015). Los nuevos rostros de la desigualdad en el siglo XXI: la brecha digital. Revista Mexicana de Ciencias Políticas y Sociales, 223, 265 - 286.

- Barrantes, G., Casas, L., \& Luengo, R (2011). Obstáculos percibidos para la integración de las TIC por los profesores de Infantil y Primaria en Extremadura. Pixel-Bit, 39, 83 - 94

- Cabero, J. (2016). Tendencias educativas para el siglo XXI. Madrid: Ediciones CEF.

- Cabero, J., \& Llorente-Cejudo, M. C. (2013). La aplicación del juicio de experto como técnica de evaluación de las tecnologías de la información (TIC). Eduweb, 7(2), 11-22.

- Cassany, D., \& Ayala, G. (2008). Nativos e inmigrantes digitales en la escuela. Participación educativa, 9, $53-71$.

- Castañeda, H. A. A. (2017). Estado actual de las competencias TIC de docentes. Puente, 9(2), 23-32.

- Castaño, C. (2008). La segunda brecha digital. Madrid: Ediciones Cátedra.

- Coll, C., Mauri, T., \& Onrubia, J. (2008). Análisis de los usos reales de las TIC en contextos educativos formales: una aproximación sociocultural. Revista electrónica de investigación educativa, 10(1), 1 - 18.

- Fernández, F.J., \& Fernández, M.J. (2016). Los docentes de la Generación Z y sus competencias digitales. Comunicar, 46, 97 - 105. https://doi.org/10.3916/C46-2016-10

- Gilbert, P. (2014). Digital literacy. NC State University. Raleigh. NC.

- Gutiérrez, A. (2008). Las TIC en la formación del profesorado. "Realfabetización" digital del profesorado. Revista interuniversitaria de formación del profesorado, 63 (22,3), 191 - 206.

- Hernández, R., Fernández, C., \& Baptista, P. (2014). Metodología de la investigación. McGraw Hill Education. Interamericana Editores.

- Ley Orgánica 1/1990, de 3 de octubre, de educación. Boletín Oficial del Estado, núm. 238, de 4 de octubre de 1990, pp. 13 a 651. https://bit.ly/2W791KI

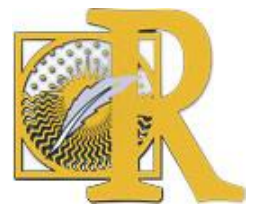

Fecha de recepción: 26-04-2019 Fecha de aceptación: 24-10-2019 
- Ley Orgánica 2/2006, de 3 de mayo, de educación. Boletín Oficial del Estado, núm. 6, de 4 de mayo de 2006, pp. 17158 a 17207. http://bit.ly/2ZDhTZI

- Ley Orgánica 8/2013, de 9 de diciembre, para la mejora de la calidad educativa. Boletín Oficial del Estado, núm. 295, de 10 de diciembre de 2013, pp. 97858 a 97921. http://bit.ly/2GAuwMf

- Llamas-Salguero, F., \& Macías Gómez, E. (2017). Formación inicial de docentes en educación básica para la generación de conocimiento con las Tecnologías de la Información y la Comunicación. Revista Complutense De Educación, 29(2), 577-593. http://dx.doi.org/10.5209/RCED.53520

- Lorenzo, J. (2005). La lectura en la generación de la Red. Jóvenes, lectura e Internet. Revista de estudios de juventud, 70, 65-79.

- Luz, C. G. M. (2018). Educación y tecnología: estrategias didácticas para la integración de las TIC. Editorial UNED.

- Mayorga, M.J., Madrid, D., \& Núñez, F. (2011). La competencia digital de los docentes. Formación y actualización en Web 2.0. Etic@net, 9(11), 213 - 232.

- Norris, P. (2001). Digital Divide, Civic Engagement, Information Poverty and the Internet Worldwide. Cambridge: Cambridge University Press.

- OECD (2015). Students, Computers and Learning: Making the connection. PISA, OECD Publishing. http://dx.doi.org/10.1787/9789264239555-en

- Plaza de la Hoz, J. (2017). Autoridad docente y Nuevas Tecnologías: cambios, retos y oportunidades. Revista Complutense de Educación, 29(1), 269-285. http://dx.doi.org/10.5209/RCED.52281

- Porlán, I. G., Espinosa, M. P. P., \& Sánchez, F. M. (2018). Competencia digital: una necesidad del profesorado universitario en el siglo XXI. RED: Revista de Educación a Distancia, 56, 1-22. http://dx.doi.org/10.6018/red/56/7

- Prensky, M. (2011). Enseñar a nativos digitales. Madrid: SM.

- Prensky, M. (2001). Digital natives, digital immigrants. On the Horizon. MCB University Press, 9(5), 1 - 6. https://doi.org/10.1108/1074812011042816

- Reygadas, L. (2008). Tres matrices generadoras de desigualdades. Pobreza humana, desigualdad y exclusión social en la ciudad del siglo XXI. México: Siglo XXI y Universidad Nacional Autónoma de México.

- Sánchez, P., Ramos, F.J. \& Sánchez, J. (2014). Formación continua y competencia digital docente: el caso de la Comunidad de Madrid. Revista Iberoamericana de Educación, 65, 91-110.

- Travieso, J.L., \& Planella, J. (2008). La alfabetización digital como factor de inclusión social: una mirada crítica. UOC Papers, Revista sobre la sociedad del conocimiento, 6, $1-8$.

- Sangrá, L. M. (2016). La nueva brecha digital docente. El futuro de las nuevas tecnologías en Primaria desde la formación del profesorado. Revista Electrónica Interuniversitaria de Formación del Profesorado, 19(2), 301-313.

- Van Dijk, J. (2006). Digital divide research, achievements and shortcomings. Poetics, 34 (4-5), 221 - 235. https://doi.org/10.1016/j.poetic.2006.05.004

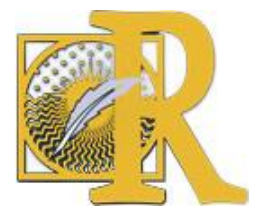

Fecha de recepción: 26-04-2019 Fecha de aceptación: 24-10-2019 